\title{
Quantitative magnetic force microscopy on perpendicularly magnetized samples
}

\author{
Hans J. Hug, B. Stiefel, P. J. A. van Schendel, A. Moser, R. Hofer, S. Martin, \\ and H.-J. Güntherodt \\ Institute of Physics, University of Basel, Klingelbergstrasse 82, CH-4056 Basel, Switzerland
}

Steffen Porthun, Leon Abelmann, and J. C. Lodder

MESA Institute, University of Twente, PoBox 217, 7500 AE Enschede, The Netherlands

Gabriel Bochi and R. C. O'Handley

Massachusetts Institute of Technology, Cambridge, Massachusetts 02139

(Received 15 September 1997; accepted for publication 27 February 1998)

\begin{abstract}
We present a transfer-function approach to calculate the force on a magnetic force microscope tip and the stray field due to a perpendicularly magnetized medium having an arbitrary magnetization pattern. Under certain conditions, it is possible to calculate the magnetization pattern from the measured force data. We apply this transfer function theory to quantitatively simulate magnetic force microscopy data acquired on a $\mathrm{CoNi} / \mathrm{Pt}$ multilayer and on an epitaxially grown $\mathrm{Cu} / \mathrm{Ni} / \mathrm{Cu} / \mathrm{Si}(001)$ magnetic thin film. The method described here serves as an excellent basis for (i) the definition of the condition for achieving maximum resolution in a specific experiment, (ii) the differences of force and force $z$-derivative imaging, (iii) the artificial distinction between domain and domain wall contrast, and finally (iv) the influence of various tip shapes on image content. (C) 1998 American Institute of Physics. [S0021-8979(98)03611-1]
\end{abstract}

\section{INTRODUCTION}

Magnetic force microscopy $(\mathrm{MFM})^{1-5}$ has become a valuable tool for imaging the stray field of complicated ferromagnetic samples ${ }^{6-15}$ and superconductors. ${ }^{16-23}$ The instrument consists of a micron-scale ferromagnetic tip attached to a flexible cantilever which scans close to the surface of the sample. The stray field emanating from the sample generates a force on the magnetic force microscope tip. The ability of the instrument to image both the magnetic stray field of the sample and sample topography ${ }^{24-26}$ allows a correlation of the measured stray field with particular surface structures. Thus it is possible to study the pinning of domain walls in ferromagnetic samples and the pinning of vortices in superconducting samples due to a structural defect observable at the surface. Other applications are the use of the stray field of the tip ${ }^{27-29}$ for modifying the magnetic state of the sample $\mathrm{e}^{22,30-32}$ or for determining the sensitivity and response of magnetic heads to localized fields. ${ }^{33-38}$

There is growing interest to move from qualitative imaging to quantitative analysis of sample properties using MFM. To this end, image analysis in reciprocal space and the use of transfer functions is now being explored. In general it is not possible to calculate a magnetization distribution from MFM data. In the special case of perpendicular magnetization, as we will show later, it is theoretically possible to use the force pattern, $F(x, y)$, to generate the magnetization pattern, $M(x, y)$, to within a constant. However due to the limited signal-to-noise ratio of a MFM force measurement, the better procedure remains to assume a magnetization pattern, calculate its field, and then calculate the force on the tip due to this field. This calculated force is then compared with the measured force data. This process is iterated until the agreement between measured and calculated force patterns is optimized. Important tasks remain including the understanding of the image formation process. This can be difficult because of the possibly strong perturbation of the sample magnetization by the magnetization of the tip, or vice versa. Generally, the MFM image formation can be divided into three categories: first, the hard magnetic case, where the magnetization of the tip and the sample remains undisturbed by the scanning process of the magnetic tip above the sample; second, the hysteresis free, soft magnetic case, where the sample or the tip magnetization is uniquely defined by the position of the tip above the sample (and the magnetic structure of the tip and the sample); ${ }^{16,21}$ and third, the intermediate case, where the magnetization of the tip and the sample influence each other and change in a discontinuous, hysteretic way. ${ }^{39}$ The ideal MFM situation, even when measuring soft samples, is a magnetically hard tip with small enough volume that the tip field is so weak that it does not perturb the sample magnetization. The small tip volume also makes higher resolution possible. However, the size of the tip volume is limited by the sensitivity of the force microscope system to detect smaller tip deflections.

In this article, we restrict the discussion to the hard magnetic case because we believe that the increased sensitivity of future scanning force microscope instrumentation allows the use of hard, low magnetic volume tips. So far, the following approaches for modeling (MFM) data have been presented. (i) In the case of simple tip and sample magnetization structures, the MFM response is often calculated analytically in direct space. ${ }^{24,40-50}$ (ii) More complex magnetization structures, including configurations with soft magnetic tips and/or samples have been treated numerically by dividing the tip 
and the sample into many subcells. ${ }^{51-56}$ However, both methods are of limited utility for many MFM applications. In the first case, complex magnetization structures cannot be described in direct space by analytic solutions, while in the second case a large amount of processor time is needed even for relatively simple cases. (iii) In contrast, an approach based on Fourier transform methods gives more insight into the basic image formation principles and is applicable to arbitrary samples and tips as long as the micromagnetic state of the tip is not influenced by the sample and vice versa (hard magnetic case). ${ }^{57}$ Schönenberger et al. ${ }^{3}$ have used the Fourier transform method to study the image formation properties of tips made from different ferromagnetic materials. In Refs. 58 and 59 the micromagnetic structure of the sample was the main point of interest while the tip was not taken into account or it was assumed to be of a rather simple structure. The first article ${ }^{58}$ discusses noise problems and image focusing, while the latter ${ }^{59,60}$ addresses the problem of the reconstruction of the sample magnetization from the measured field data. All these approaches calculate the force on the magnetized tip in the stray field of the sample.

Alternatively, the modeling of MFM data has been accomplished using the force reciprocity principle. ${ }^{61}$ The authors calculate the force acting on the magnetization of the sample produced by the stray field of the tip rather than vice versa. This allows the role of the tip in the imaging process to be represented in a particularly simple fashion.

In this article we outline a transfer function theory appropriate to MFM and demonstrate its power for quantitative analysis of MFM images. In Sec. II we use a Fourier transform method to calculate the stray field of an arbitrarily complex magnetization. ${ }^{62}$ We find transfer functions that allow the determination of the Fourier components of the field from those of the magnetization and those of the force from those of the field by simple multiplication. The capabilities and the limits of the inversion of these transfer functions are discussed. The enhancement of the resolution of an MFM image [if defined as the full width at half maximum (FWHM) of the force image of a transition of the magnetization] and the reconstruction of the magnetization pattern from the measured data are shown to play an important role in MFM experiments. In Sec. III, we first describe the experimental conditions that must be followed in order to allow proper interpretation of MFM data. We then apply our theory to the analysis of magnetic force microscopy data acquired on a $\mathrm{CoNi} / \mathrm{Pt}$ multilayer and on an epitaxially grown $\mathrm{Cu} / \mathrm{Ni} / \mathrm{Cu} / \mathrm{Si}(001)$ magnetic thin film.

\section{THEORY}

One challenge in interpreting MFM images is that threedimensional spatial integration is necessary to get to the field from the magnetization and the force from the field: $M(x, y) \rightarrow \mathbf{H}(x, y, z) \rightarrow \mathbf{F}(x, y, z)$. The more efficient way is to Fourier transform those fields and make use of the fact that their Fourier transforms are simply related by multiplicative functions called transfer functions: $M\left(k_{x}, k_{y}\right)$ $\rightarrow \mathbf{H}\left(k_{x}, k_{y}, z\right) \rightarrow \mathbf{F}\left(k_{x}, k_{y}, z\right)$. In this section we summarize those aspects of Fourier transfer functions that are important for quantitative understanding of MFM images. We first assume a perpendicular sample magnetization distribution, $M_{z}(x, y)$, which is uniform over the film thickness, $h$. The magnetization, $M_{z}(x, y)$, gives rise to surface charge distributions ${ }^{63}$ at the top surface $[+\sigma(x, y), z=0]$ and the bottom surface $[-\sigma(x, y), z=-h]$. The magnetic field above the sample is produced by these charges only. If necessary, bulk charges can easily be introduced, for instance by layering the magnetic film into sheets of surface charges. Following $^{64,65}$ we perform the calculation in the twodimensional (2D) spatial frequency domain, using the Fourier description of the surface charges at the top and the bottom of the film

$$
\begin{aligned}
& A_{M}(\mathbf{k})=\int_{-\infty}^{\infty} \sigma(\mathbf{r}) \cdot e^{i \mathbf{k r}} \cdot d \mathbf{r} \\
& \text { with } \mathbf{r}=(x, y), \quad \mathbf{k}=\left(k_{x}, k_{y}\right) \text { and } k=\sqrt{k_{x}^{2}+k_{y}^{2}} .
\end{aligned}
$$

As is common in magnetostatic calculations, we define a magnetostatic potential, $\phi(x, y, z)$ as

$$
\mathbf{H}(x, y, z)=-\operatorname{grad} \phi(x, y, z) .
$$

The magnetostatic potential must be a solution of the Laplace equation in the free space outside the magnetic sample. A general solution of the Laplace equation, which vanishes as the distance, $z$, goes to infinity, is

$$
\phi(x, y, z)=\int_{0}^{\infty} A_{\phi}(\mathbf{k}) \cdot e^{i \mathbf{k} \cdot \mathbf{r}} \cdot e^{-\sqrt{k_{x}^{2}+k_{y}^{2}}} d \mathbf{k} .
$$

Using the boundary condition

$$
\left.\frac{\partial \phi}{\partial z}\right|_{z=+0}=-\frac{1}{2} M(x, y),
$$

the Fourier components of the magnetization, $A_{M}(\mathbf{k})$, and those of the magnetostatic potential, $A_{\phi}(\mathbf{k})$, used in Eq. (4) are related as

$$
A_{\phi}(\mathbf{k})=\frac{A_{M}(\mathbf{k})}{2 \sqrt{k_{x}^{2}+k_{y}^{2}}} .
$$

Equation (6) expresses in the simplest form, the use of a transfer function, $(2 k)^{-1}$, to relate the Fourier transforms of the sample magnetization and the magnetostatic potential. Its utility will become clearer as we proceed.

Using Eqs. (3), (4), and (6) we can easily find the relation between the Fourier components of the stray field, $A_{H_{x, y, z}}^{z, h}(\mathbf{k})$, and those of the magnetization [Eq. (1)],

$$
\begin{aligned}
A_{H_{x, y}}^{z, h}(\mathbf{k}) & =i \cdot \frac{e^{-k z}\left(1-e^{-k h}\right) k_{x, y}}{2 k} \cdot A_{M}(\mathbf{k}) \\
& =\mathrm{HTF}_{x, y}(\mathbf{k}) \cdot A_{M}(\mathbf{k}), \\
A_{H_{z}}^{z, h}(\mathbf{k}) & =\frac{e^{-k z}\left(1-e^{-k h}\right)}{2} \cdot A_{M}(\mathbf{k})=\operatorname{HTF}_{z}(\mathbf{k}) A_{M}(\mathbf{k}),
\end{aligned}
$$

where the $\mathrm{HTF}_{i}$ are called the field transfer functions. Note that the factor $\left(1-e^{-k h}\right)$ originates from the magnetic surface charge density at the lower surface of the film. The 


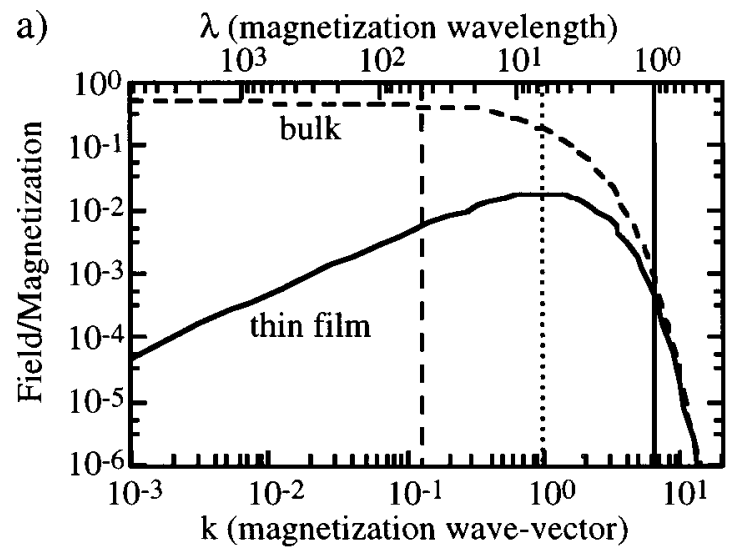

b)

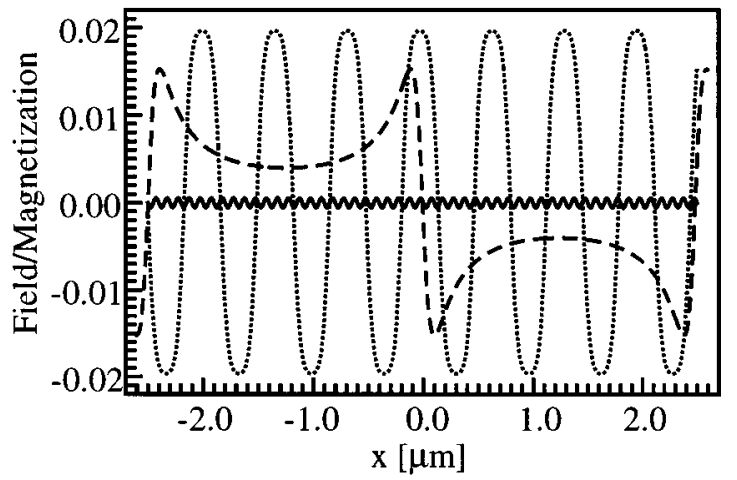

FIG. 1. (a) The field-transfer function (HTF), which is the ratio of the Fourier components of the field to those of magnetization, is plotted as a function of the wave vector, $k=2 \pi / \lambda$ (and wavelength, $\lambda$ ). The dashed curve represents the HTF for a bulk sample and the solid curve is the HTF for a thin film sample $(h=0.1)$. In both cases the distance from the sample is $z=1$ and the sample magnetization is $M=1$. Clearly the HTF for the bulk sample reaches its maximum value of 0.5 for the large magnetization periods (small wave vectors) and decays exponentially at short magnetization periods. In contrast, the HTF of the thin film sample reaches its maximum value for $\lambda=2 \pi h / \ln [(z+h) / z]$ (dotted line in b) and rolls off for short and long magnetization periods (dashed and solid line in b). (b) The stray field of a square magnetization pattern in a perpendicular thin film (magnetization up and down) is plotted as a function of the lateral distance, $x$ in $\mu \mathrm{m}$, for three domain periods. The sample thickness is $10 \mathrm{~nm}$, and the tip-to-sample distance is $100 \mathrm{~nm}$. The dashed line is the field for a magnetization wavelength of $5000 \mathrm{~nm}(\lambda=50)$, the dotted line for $659 \mathrm{~nm}\left(\lambda=6.59=\lambda_{\max }\right)$, and the solid line for $100 \mathrm{~nm}(\lambda=1)$.

magnetic stray field at the position, $\mathbf{r}$, a distance, $z$, above the surface of the film can be calculated by the reverse transform

$$
\begin{aligned}
& H_{x, y}(\mathbf{r}, z)=\frac{1}{4 \pi^{2}} \int_{-\infty}^{\infty} A_{H_{x, y}^{z, h}}^{z, i \mathbf{r r}} \cdot d \mathbf{k}, \\
& H_{z}(\mathbf{r}, z)=\frac{1}{4 \pi^{2}} \int_{-\infty}^{\infty} A_{H_{z}}^{z, h} e^{-i \mathbf{k r}} \cdot d \mathbf{k} .
\end{aligned}
$$

In Fig. 1(a), we plot the field transfer function (HTF) which, by Eq. (8), is equal to the ratio of the Fourier components of the field and the magnetization. The HTF is plotted for a bulk sample (thickness, $h=\infty, z=1$ ) and a thin film sample (thickness, $h=0.1, z=1$ ) in dimensionless units. These transfer functions, when multiplied by the Fourier coefficients of the magnetization distribution, give the Fourier coefficients of the field. Figure 1(b) shows the $x$-dependence of

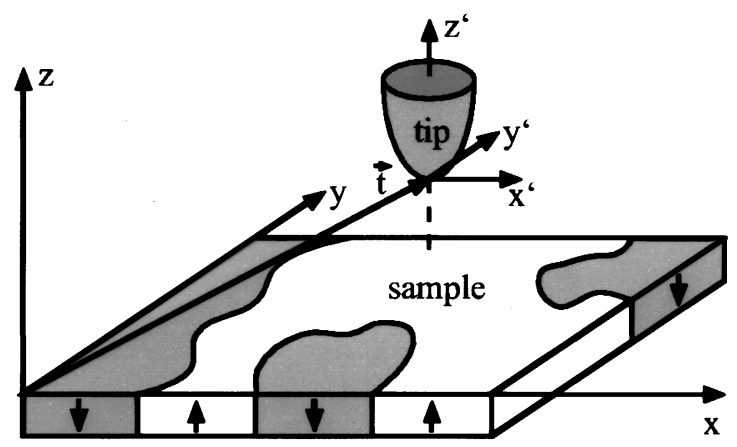

FIG. 2. The sample coordinate system is attached to the sample surface ( $z$ $=0)$. The primed coordinate system is attached at the tip located at the position $\left(t_{x}, t_{y}, t_{z}\right)$.

the field, $H_{z}$, for square magnetization distributions (many Fourier components) in a thin film for three different magnetization periods. For a long magnetization period (e.g., 5 $\mu \mathrm{m}$, dashed line) the fundamental spatial frequency of the magnetization is multiplied by a small HTF value. Hence the field above the center of the domain is weak. The higher spatial frequency components of the magnetization which occur near the domain walls are amplified by a larger HTF so the field there is larger. (In earlier articles this strengthening of the field above a sharp change in magnetization was often called domain wall contrast.) Note that the largest stray field is generated for a magnetization wavelength given by

$$
k=\frac{\ln \left(\frac{z+h}{z}\right)}{h} .
$$

This field is shown by the dotted line in Fig. 1(b). In this case, the fundamental Fourier component of the magnetization occurs at the peak in the HTF so the largest component of the field is at the fundamental period of the magnetization. For very small magnetization periods [solid line in Fig. 1(b)] the exponential decay of the field-transfer function leads to a strong attenuation of the stray field. In this case, good resolution can be obtained only if the MFM has very high sensitivity. Thus we can see that the HTF is a function that generates fields of different strength from magnetization features of different wavelength.

Once the stray field of a magnetic sample is known, the force on the MFM tip is found by integrating the product of the field and the magnetic charge distribution of the tip over the tip volume. We define a tip volume charge distribution, $\rho_{\text {Tip }}$, and a tip surface charge distribution, $\sigma_{\text {Tip }}$, as

$$
\begin{aligned}
& \rho_{\text {Tip }}\left(x^{\prime}, y^{\prime}, z^{\prime}\right)=\operatorname{div} \mathbf{M}\left(x^{\prime}, y^{\prime}, z^{\prime}\right) \forall\left(x^{\prime}, y^{\prime}, z^{\prime}\right) \in V_{\text {Tip }}, \\
& \sigma_{\text {Tip }}\left(x^{\prime}, y^{\prime}, z^{\prime}\right)=\mathbf{M}_{\text {Tip }} \cdot \mathbf{n} \forall\left(x^{\prime}, y^{\prime}, z^{\prime}\right) \in A_{\text {Tip }},
\end{aligned}
$$

where the origin of the primed coordinate system is attached to the end of the tip (Fig. 2). Then the $z$ component of the force on the tip located at the position $(\mathbf{r}, z)$ becomes 


$$
\begin{aligned}
F_{z}(\mathbf{r}, z)= & \mu_{0} \cdot \int_{V} \rho_{\mathrm{Tip}}\left(\mathbf{r}^{\prime}, z^{\prime}\right) H_{z}\left(\mathbf{r}+\mathbf{r}^{\prime}, z+z^{\prime}\right) d V^{\prime}+\mu_{0} \\
& \cdot \oint_{A} \sigma_{\mathrm{Tip}}\left(\mathbf{r}^{\prime}, z^{\prime}\right) H_{z}\left(\mathbf{r}+\mathbf{r}^{\prime}, z+z^{\prime}\right) d A^{\prime}
\end{aligned}
$$

Alternatively, the force may be calculated by taking the $z$ derivative of the magnetostatic energy of the tip in the stray field of the sample (see appendix).

The force can also be calculated in Fourier space. The Fourier descriptions of the force and the field,

$$
F_{z}(\mathbf{r}, z)=\frac{1}{4 \pi^{2}} \int_{-\infty}^{\infty} A_{F_{z}}^{z, h} e^{-i \mathbf{k r}} \cdot d \mathbf{k}
$$

and

$$
H_{z}\left(\mathbf{r}+\mathbf{r}^{\prime}, z+z^{\prime}\right)=\frac{1}{4 \pi^{2}} \int_{-\infty}^{\infty} A_{H_{z}}^{z, h} e^{-i \mathbf{k}\left(\mathbf{r}+\mathbf{r}^{\prime}\right)} e^{-k z^{\prime}} d \mathbf{k},
$$

can be inserted in Eq. (12). Then we find the relation between the Fourier components as

$$
A_{F_{z}}^{z, h}(\mathbf{k})=A_{H_{z}}^{z, h}(\mathbf{k}) \cdot \operatorname{FTF}(\mathbf{k})
$$

with

$$
\begin{aligned}
\operatorname{FTF}(\mathbf{k})= & \mu_{0} \cdot \int_{V} \rho_{\mathrm{Tip}}\left(\mathbf{r}^{\prime}, z^{\prime}\right) e^{-k z^{\prime}} e^{i \mathbf{k} \mathbf{r}^{\prime}} d V^{\prime}+\mu_{0} \\
& \cdot \oint_{A} \sigma_{\mathrm{Tip}}\left(\mathbf{r}^{\prime}, z^{\prime}\right) e^{-k z^{\prime}} e^{i \mathbf{k} \mathbf{r}^{\prime}} d A^{\prime}
\end{aligned}
$$

Finally we define the tip-transfer function, TTF (k), as the product of the field-transfer function and the force-transfer function (see Fig. 5):

$$
\operatorname{TTF}(\mathbf{k})=\operatorname{HTF}(\mathbf{k}) \operatorname{FTF}(\mathbf{k}) .
$$

For a given image size (set of $k$ vectors) the two integrals in Eq. (16) need to be calculated only once to be applied to an arbitrary magnetization pattern. ${ }^{66}$ Note that the force-transfer function reflects the magnetic microstructure of the MFM tip only, whereas the field-transfer function, HTF $(\mathbf{k})$ is determined by the magnetic sample (bulk, thin film, multilayer, etc.).

The three transfer functions relevant to MFM image analysis have now been defined. We now show the power of these functions by demonstrating their use in defining the field of more complex sample or tip charge distributions. From Eq. (8), the Fourier components of the field above a single surface charge distribution, $\sigma(\mathbf{k})=\sigma(\mathbf{k}, z=0)$ is

$$
A_{H_{z}}^{\sigma, z}=\operatorname{HTF}(\mathbf{k}) \cdot \sigma(\mathbf{k})=\frac{e^{-k z}}{2} \sigma(\mathbf{k}) .
$$

The field above a double layer, $\sigma(\mathbf{k})=\sigma(\mathbf{k}, z=0)-\sigma(\mathbf{k}, z$ $=-h)$, is simply

$$
\begin{aligned}
A_{H_{z}}^{\sigma^{ \pm}, z} & =\operatorname{HTF}(\mathbf{k}) \cdot\left[\sigma(\mathbf{k})-\sigma(\mathbf{k}) \cdot e^{-k h}\right] \\
& =\frac{e^{-k z}\left[1-e^{-k h}\right]}{2} \sigma(\mathbf{k}) .
\end{aligned}
$$

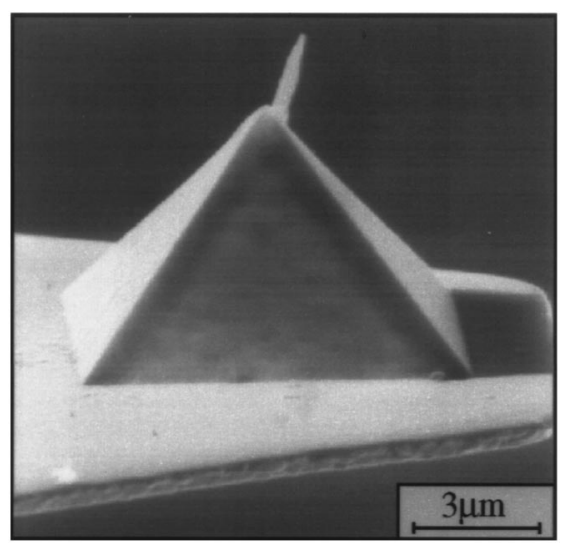

FIG. 3. To obtain a tip with a well defined direction of the magnetization we have used an electron microscope to grow a deposition tip on a standard pyramid tip of a commercially available cantilever. The needlelike tip was intentionally grown at an angle of approximately twelve degrees to the axis of the pyramid tip, so that it is perpendicular to the surface of the sample. The tip is made sensitive to magnetic stray fields by a 25 -nm-thick Co film which was deposited on the side of the needlelike tip by thermal evaporation.

By extension, the Fourier components of the field above a continuous, $z$-dependent distribution of charges are given by

$$
\begin{aligned}
A_{H_{z}}^{\sigma\left(z^{\prime}\right), z}(\mathbf{k}) & =\int \operatorname{HTF}(\mathbf{k}) \cdot \sigma\left(\mathbf{k}, z^{\prime}\right) e^{-k z^{\prime}} d z^{\prime} \\
& =\frac{1}{2} \cdot \int e^{-k z} \sigma\left(\mathbf{k}, z^{\prime}\right) e^{-k z^{\prime}} d z^{\prime} .
\end{aligned}
$$

It is not possible to make a simple statement of the real space field above an arbitrary charge distribution because each $k$ value of $\sigma(k)$ decays with distance at a different rate.

Note that Eq. (16) is of the same form as Eq. (20) for $z=0$. Thus the force-transfer function [Eq. (16)] is the Fourier transform of the stray field of the tip in the plane $\left(x^{\prime}, y^{\prime}, z^{\prime}=0\right)$.

In this work we restrict our calculations of the forcetransfer function, FTF (k), to a long, thin, slablike tip (see Fig. 3), with

(i) its bottom and top surface area given by, $\left(b_{x} b_{y}\right)$,

(ii) a homogeneous magnetization directed along its long axis, $l$, and

(iii) the tip oriented perpendicular to the surface of the sample.

Using Eq. (16) we find for the FTF (k)

$$
\operatorname{FTF}(\mathbf{k})=-\frac{4 \mu_{0} M_{\mathrm{Tip}}}{k_{x} k_{y}} \sin \left(\frac{k_{x} b_{x}}{2}\right) \sin \left(\frac{k_{y} b_{y}}{2}\right)\left(1-e^{-k l}\right) .
$$

In Fig. 4(a) we have plotted Eq. (21) as a function of the wave vector, $k_{x}$, in one dimension with a tip length $l=10$, a bottom surface edge length, $b=0.1$, and $\mu_{0}=1$. The FTF is flat over a large range of wave vectors and decays for small and large wave vectors. The decay for small wave vectors (large magnetization wavelengths) is due to the finite length of the tip (dipole tip behavior) through the last factor in Eq. (21). The decay for large wave vectors is caused by the 
a)

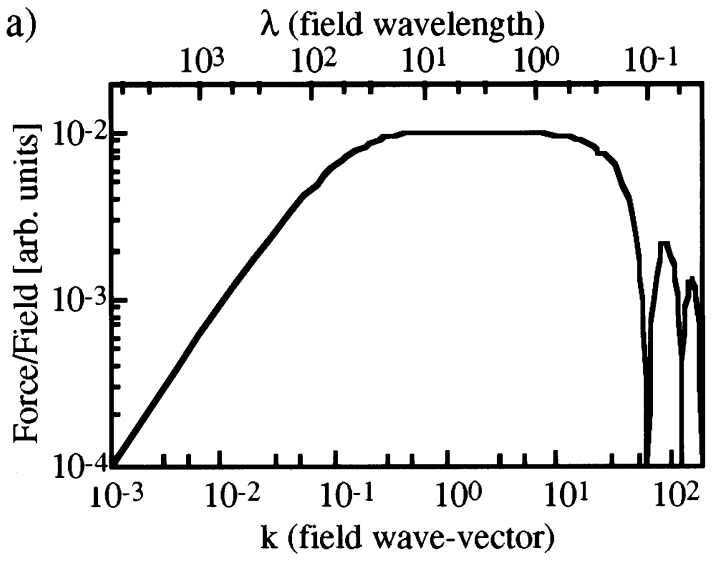

b)

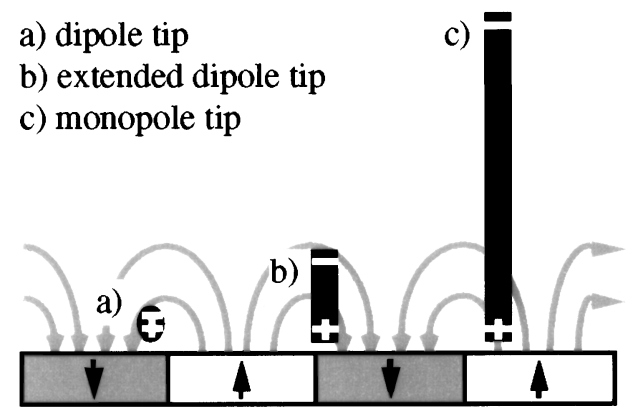

FIG. 4. (a) The force-transfer function (FTF) which is the ratio of the Fourier components of the force to those of the field, is plotted as a function of the wave-vector, $k=2 \pi / \lambda$ (and wavelength, $\lambda$ ) for a slablike tip [Eq. (21)]. Tip length, $l=10$; size of bottom and top surface surface, $b=0.1$; tip magnetization, $M_{\text {Tip }}=1$. (b) Various tip lengths in a field emanating from a periodic magnetization pattern. The force on the dipole tip (a) is proportional to the $z$ derivative of the field, whereas the force on the monopole tip (c) is proportional to the stray field (tip length much larger than the decay constant of the stray field). The force on the elongated-dipole tip (b) can be calculated as the force difference between the lower, positive charge, and the upper negative charge.

$1 / k_{x} k_{y}$ dependence in Eq. (21). Further the sine terms introduce a series of zeros in the force-transfer function related to the bottom area of the tip, $b_{x} b_{y} .{ }^{67}$ The size of the bottom surface of the tip, $\left(b_{x} b_{y}\right)$, thus sets a natural limit to the resolution of the stray field. The measured force is proportional to the stray field as long as the length of the tip is larger than the decay length of the field, $e^{-k l} \ll 1$, [see appendix, Eqs. (A5), (A6), and Fig. 4(b)]. If the bottom surface of the tip is much smaller than the shortest wavelengths of the magnetic surface charge distribution, $k_{x} b_{x} \ll 1$ and $k_{y} b_{y} \ll 1$ (the flat region of the FTF, Fig. 4(a), should be extended to smaller $k$ values.), then the force-transfer function, FTF [Eq. (21)], can be approximated by a point charge, $q_{m}$

$$
\operatorname{FTF}(\mathbf{k}) \approx-\mu_{0} M_{\text {Tip }} b_{x} b_{y}=q_{m} .
$$

It is important to note the analogy between the transferfunction analysis of MFM and magnetic recording theory. An MFM tip resembles a single pole head used in perpendicular magnetic recording. (i) In the $e^{-k z}$ term we see the same behavior as described by head-to-medium distance loss. (ii) In the TTF [Eqs. (21) and (17)] we recognize the sinusoidal functions to give a behavior like the inductive head gap loss [FTF (k)]. (iii) Contained in the HTF [Eq. (8)]

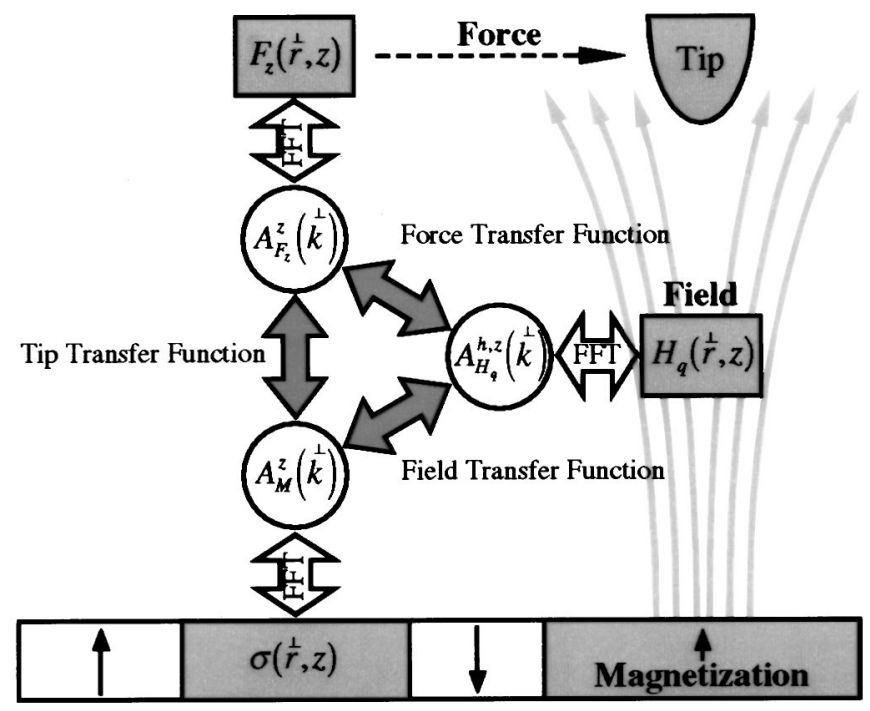

FIG. 5. The Fourier components of the force, $F_{z}$, on the MFM tip are calculated either directly from those of the magnetic surface charges, $A_{M}(\mathbf{k})$, by means of the tip-transfer function [TTF, Eq. (17)] or indirectly from the Fourier components of the stray field, $A_{H z}^{h, z}$, by means of the forcetransfer function [FTF, Eq. (16)], where the stray field is calculated by the field-transfer function, $\left[\mathrm{HTF}_{z}\right.$, Eq. (8)].

we see a term $1-e^{-k h}$, identical to that used to describe the effects of recorded signal depth, $h$, on field strength, namely the film thickness loss. ${ }^{68}$

In conclusion (see Fig. 5) the force on the MFM tip can be calculated directly from the magnetic surface charges on the tip by means of the tip-transfer function [TTF, Eq. (17)] or, alternatively from the stray field by means of the forcetransfer function [FTF, Eq. (16)]. The stray field is calculated from the Fourier component of the sample magnetization by the field-transfer function, $\left[\mathrm{HTF}_{z}\right.$, Eq. (8)]. With these equations it is further possible to calculate many important quantities.

(1) The stray field can be determined from the force image. This process is limited by the cutoff frequency of the force-transfer function [Fig. 4(a) $]^{69}$ or by the force sensitivity of the MFM (the rapid decay of the force-transfer function for small wave vectors may lead to a cutoff given by the limited sensitivity of the force microscope instrumentation rather than by the finite size of the tip).

(2) One can calculate the stray field at a smaller tip-tosample distance, $z$, from data acquired at a larger tip-tosample distance, $Z$, by

$$
A_{H_{z}}(\mathbf{k})=A_{H_{Z}}(\mathbf{k}) \cdot e^{k(Z-z)} \text {. }
$$

Note that this process is limited by the signal-to-noise ratio of the measurement.

(3) The sample surface charge distribution can be reconstructed from the stray field [set $z=0$ in Eq. (23)]. Note that in the case of perpendicular magnetization, the surface charge distribution is equal to one half of the magnetization. Again, the process is limited by the signal-tonoise ratio of the field or force data.

(4) The $z$ derivative of the field can be determined from the 
field or force by

$$
A_{z \text { derivative }}(\mathbf{k})=A_{\text {Field }}(\mathbf{k}) \cdot(-k) \text {. }
$$

Note that operation modes which measure the force derivative amplify the high frequency Fourier components and seem to be well suited for high-resolution MFM. However, dynamic mode MFM images are more difficult to interpret (see discussion in next section).

(5) The stray field of the tip, $H_{z, \text { Tip }}\left(\mathbf{r}^{\prime}, z^{\prime}\right)$ is equal to $2 \mu_{0}$ times the force-transfer function [Eq. (16)] which is used to calculate the Fourier components of the force, $A_{F_{x}}$, from those of the field, $A_{H_{z}}^{z, h}$.

With the transfer-function approach we have found an efficient way to calculate the Fourier components of the force and the stray field from those of the magnetization. A stray field or force calculation becomes similar to a regular Fourier-filtering procedure. The method described here is easily implemented using todays high-level-language image processing software packages, e.g., ${ }^{70,71}$ which allow efficient handling of the Fourier transformation of large matrices of real numbers.

\section{APPLICATION TO MFM MEASUREMENTS}

In this section we illustrate the application of the MFM transfer function theory developed in Sec. II. New quantitative analysis is presented for magnetic domain images taken on perpendicular $\mathrm{CoNi}$ magnetic recording thin films and ultrathin $\mathrm{Cu} / \mathrm{Ni} / \mathrm{Cu}$ films.

The present results were taken at room temperature and in air using a new, sophisticated fiber-optical scanning force microscope (SFM) which was built for low temperature and UHV use. ${ }^{72}$ The small size of the instrument (diameter: 40.4 mm, length: $120 \mathrm{~mm}$ ) and the thermally well compensated construction make this instrument particularly suited to reproducibly perform MFM experiments using a fixed set of operation parameters. The microscope can be used in dc and ac scanning modes of force microscopy (see article about MFM in Ref. 73). Although the ac modes have the advantage of higher sensitivity for short wavelength Fourier components [Eq. (24)], the quantitative interpretation of the data is difficult. In the following we discuss some of the most common difficulties of the interpretation of images acquired in the ac-operation modes.

In the ac mode the relative frequency shift, $\delta \omega / \omega$, in cantilever resonance is commonly assumed to be proportional to the force derivative by

$$
\frac{\delta \omega}{\omega_{0}}=\frac{1}{2 c} \frac{\partial F_{z}}{\partial z},
$$

where $c$ is the force constant of the cantilever and $\omega_{0}$ is the resonance frequency of the free cantilever. However, Eq. (25) is true only under three conditions: first, the damping of the cantilever oscillation is constant throughout the image, second, the oscillation amplitude of the cantilever is small compared to the average tip-to-sample distance and third, the tip-to-sample distance is constant.

However, using microfabricated cantilevers in air, we often find that the damping strongly depends on the local topography. ${ }^{74}$ Topographic features or even cantilever deflections caused by magnetic forces are then easily misinterpreted as force derivatives using Eq. (25). Further, the amplitude of the cantilever oscillation is a large fraction of the tip-to-sample distance in many experiments ${ }^{75-83}$ because the sensitivity depends linearly on the oscillation amplitude. The minimum detectable force $z$ derivative is given by ${ }^{84}$

$$
F_{z \min }^{\prime}=\frac{1}{A} \sqrt{\frac{2 c k_{B} T \Delta \nu}{Q \omega_{0}}},
$$

where $A$ is the oscillation amplitude, $k_{B}$ is the Boltzmann constant, $T$ is the temperature, $\Delta \nu$ is the measurement bandwidth, and $Q$ is the sharpness of the cantilever resonance. Finally, many groups have operated their instruments in a constant frequency shift (force derivative) mode. In order to have the feedback loop stable (see article about MFM in Ref. 73), it is necessary to apply an electric field between the tip and the sample in order to keep the second $z$ derivative of the force from changing its sign. This mode allows stable MFM operation even with relatively simple instrumentation, often lacking mechanical and thermal stability. However, such an operation mode leads to a nonconstant tip-to-sample distance. An elegant solution to this problem was presented by Schönenberger. ${ }^{25} \mathrm{He}$ uses the second harmonic generated by an oscillating electric field applied between the tip and the sample to keep the tip-to-sample distance constant. Then the change of the cantilever resonance frequency reflects the magnetic signal.

In the experiments presented in this work we have used a static MFM operation mode (the details of this operation mode have been described in previous work). ${ }^{26,85}$ The static mode is used to avoid the above problems and to make a quantitative interpretation possible. We do not use feedback to control the tip-to-sample distance but simply add a linear and quadratic part of the $x$ - and $y$-scan signal to the $z$ piezo in order to compensate for the sample slope and the nonlinearity in the scan-piezo movement. Using topographical measurements to find the best fitting parameters, the tip finally scans on a "least square deviation paraboloid." With the presently used tip-to-sample distances around $100 \mathrm{~nm}$ and topographical features typically $10 \mathrm{~nm}$ in height, the variation of the tip-to-sample distance can be neglected. We currently reach a force resolution of $1 \mathrm{pN}$ giving a reasonable signal-to-noise ratio of 26-34 dB.

In all experimental examples discussed below we have used a tip produced by $e$ beam induced deposition. ${ }^{86-88}$ The needlelike tip was grown on a commercially available cantilever at an angle of approximately twelve degrees to the axis of the pyramid tip, so that the needle is perpendicular to the surface of the sample. The tip is made sensitive to magnetic stray fields by a 15-25-nm-thick Co film which was deposited on the side of the tip by thermal evaporation (Fig. 3).

A first application of the MFM transfer function theory is the enhancement of the sharpness of an MFM image. In realistic MFM measurements, the minimum tip-to-sample distance is limited by the snap-to-contact distance of the cantilever. $^{26,89}$ When the sample is brought towards the tip, the cantilever deflection becomes unstable when the attractive force $z$ derivative becomes larger than the force constant 


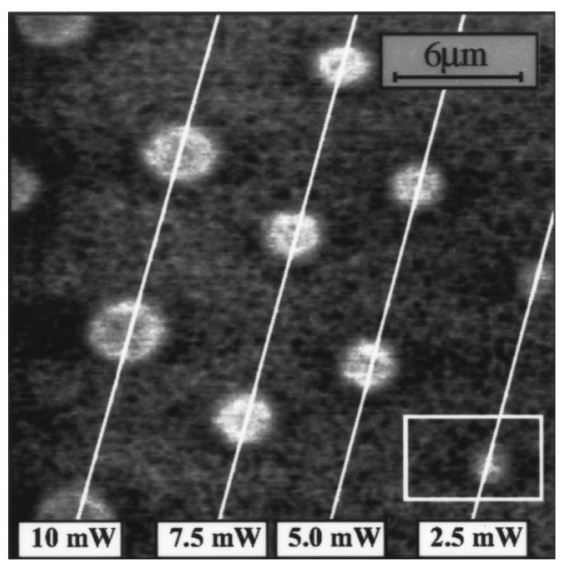

FIG. 6. A laser beam has been used to magneto-optically write bits in a multilayer, $20 \times\left(5 \AA_{C_{0}} \mathrm{Ni}_{50} / 5 \AA \mathrm{Pt}\right)$. The bits were written with laser pulses of $0.24 \mu$ s duration and powers of $2.5,5,7.5$, and $10 \mathrm{~mW}$ (Fig. 6, top). The bits are not fully saturated (subdomains exist) due to the low field applied during writing [Fig. 7(a)].

of the cantilever. At this point the cantilever snaps to contact, making a measurement of small magnetic forces impossible. $^{4,26}$

One of our experiments was performed on magnetooptically written bits in a $20 \times\left(5 \AA \mathrm{Co}_{50} \mathrm{Ni}_{50} / 5 \AA \mathrm{Pt}\right)$ multilayer. Due to the low squareness of the hysteresis loop, this material splits up into small domains which are still visible outside the bits. The bits were written with laser pulses of $0.24 \mu$ s duration and powers of 2.5, 5, 7.5, and $10 \mathrm{~mW}$ (Fig. 6 ). Note that the bits are not fully saturated due to the low field applied during writing [Fig. 7(a)]. It has been pointed out that such a sample may serve as a reference to test the resolution of magnetic force microscopes and/or MFM tips but it is not suited as a recording material..$^{90}$

With Figs. 7(a)-7(c) we show that the resolution (if defined as the sharpness of transitions) can be enhanced by using Eq. (23) as a Fourier-filtering function. We calculate the image at a reduced tip-to-sample distance of $20 \mathrm{~nm}$ [Fig. 7(b)] from data acquired at a tip-to-sample distance of $74 \mathrm{~nm}$ [Fig. 7(a)]. In Fig. 7(c) we plot the field-transfer functions for $z=74.2$ and $z=20 \mathrm{~nm}$ as a function of the magnetization wavelength, $\lambda$, rather than as a function of the wave vector, $k=2 \pi / \lambda$, [Fig. 1(a)]. The figure clarifies that a strong amplification of the small wavelength Fourier components is achieved. At a magnetization period of $100 \mathrm{~nm}$, i.e., a domain size of $50 \mathrm{~nm}$ (dotted line), the gain becomes 30. To avoid an amplification of the high frequency noise we first low-pass filter the data to suppress the amplification of wavelengths below $50 \mathrm{~nm}$ (Note that a cutoff wavelength of 50 $\mathrm{nm}$ is justified because the distance between two measurement points is $30 \mathrm{~nm}$.). Note that no information is lost as the tip-to-sample distance is increased as long the information remains above the noise level. ${ }^{91,92}$ However, in practice the sharpening is limited by the signal-to-noise ratio of the original data.

As indicated in the above discussion, a definition of the resolution relying on the measured width of a sharp transition of the magnetization is not suitable. The high frequency Fourier components are suppressed more strongly than the
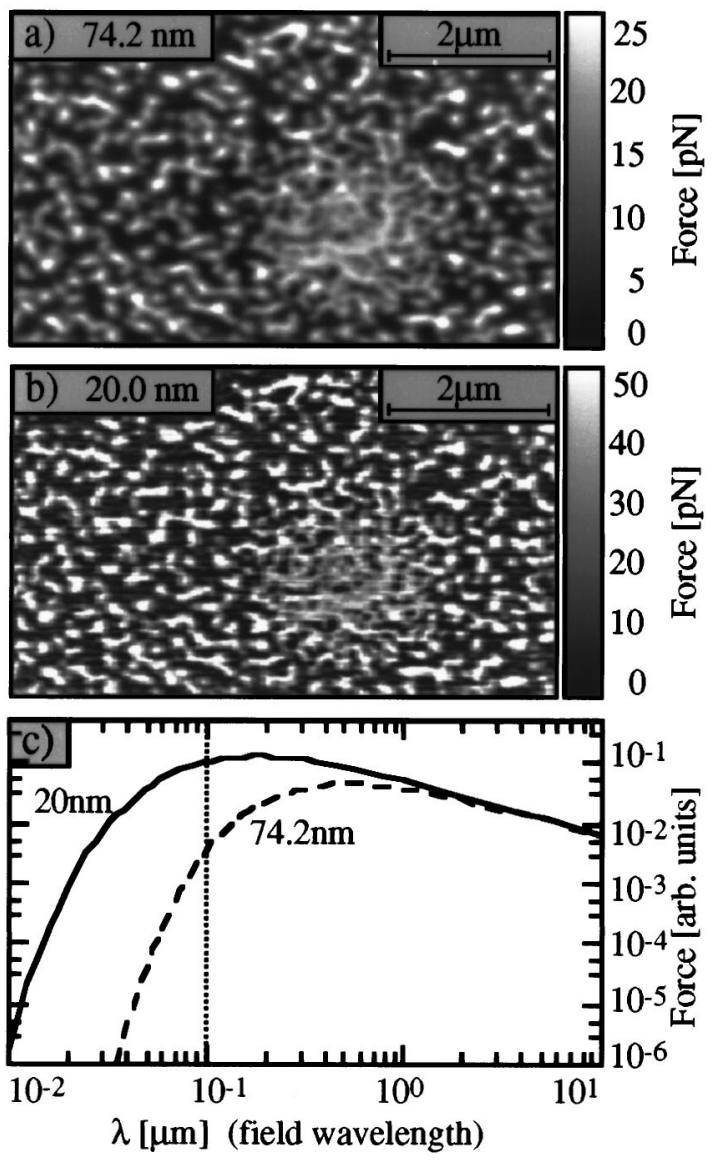

FIG. 7. Close up image of one bit from Fig. 6. The sharpness of the transitions can be enhanced by calculating the image at a reduced tip-to-sample distance of $20 \mathrm{~nm}$ (b) from data acquired at $74 \mathrm{~nm}$ (a). This process is limited by the signal-to-noise ratio of the MFM measurement performed at $74.2 \mathrm{~nm}$. (c) The field-transfer functions for the tip-to-sample distances $z$ $=74.2$ (dashed line) and $20 \mathrm{~nm}$ (solid line) are plotted as functions of the magnetization wavelength, $\lambda$ (sample thickness, $h=20 \mathrm{~nm}$, sample magnetization, $M_{\text {Sample }}=1$ ). A strong amplification of the high frequency Fourier components is achieved by measurement or simulation at small tip-tosample distances. At a magnetization period of $100 \mathrm{~nm}$ (domain size of 50 $\mathrm{nm})$ the gain becomes 30 .

low frequency ones [Eq. (8)] but can be recovered in some cases. Further, a high frequency variation of the magnetization which is overlayed on a low frequency variation of the same amplitude is easily missed in the MFM image. Without the presence of a low frequency Fourier component, the high frequency one would have been easily visible.

We suggest not to define the resolution as the sharpness of a transition but as the wavelength where the signal-tonoise ratio becomes $\sqrt{2}(3 \mathrm{~dB})$. Note that for a finite tip length and/or for a finite sample thickness there is also a long wavelength limit. However this limit is generally not important for MFM experiments. The (lateral) resolution depends on the sample properties, the tip-to-sample distance, $z$, the size and type of the tip (force-transfer function), and the minimal measurable force (vertical resolution) or force derivative of the force microscope instrumentation used. The resolution depends on sample properties through the magnitude and direction of the magnetization and the type of sample (bulk sample or thin film with thickness, $h$ ). A stronger stray field will generally allow a higher resolution. The 

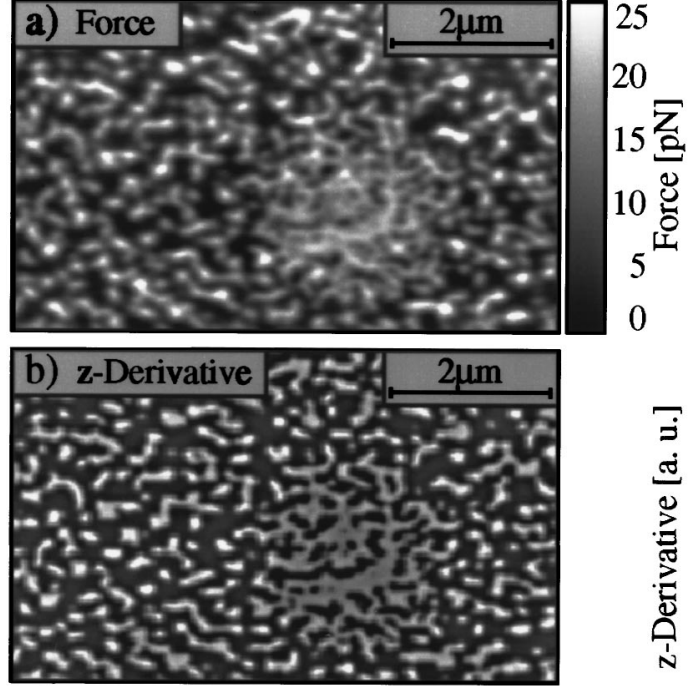

FIG. 8. In the dynamic MFM operation modes the high frequency Fourier components are enhanced relative to those measured in the static mode [image (a)]. The gradient image (b) was calculated using Eq. (24). The tip-to-sample distance, $z$, is $74 \mathrm{~nm}$; the film thickness, $h$ is $20 \mathrm{~nm}$.

role of the tip is more evident. The resolution depends on the tip size and the magnetic charge at the bottom surface. Further, an elongated dipole tip prevents an early role off of the force transfer function at small wave vectors. Finally, the force (or force derivative) resolution of the microscope might be responsible for an early cutoff of the tip-transfer function. The lateral resolution of an MFM experiment may be limited by the vertical resolution (or the signal-to-noise ratio) of the microscope rather than by the tip. To find the maximum achievable resolution, it becomes necessary to:

(1) calculate the tip-transfer function for the given tip/ sample configuration,

(2) determine the minimum measurable force of the microscope,

(3) compare the minimum detectable force (microscope sensitivity) with the tip-transfer function to determine the nature of the limit (tip or distance limited).

From a resolution point of view it is interesting to compare data acquired in the static and dynamic measurement mode. Figure 8(a) shows an MFM image acquired in the static operation mode and the negative if a simulation of a dynamic mode MFM image [Fig. 8(b)] uses Eq. (24) to calculate the force $z$ derivative image. At first glance, the images look very similar. However, in the $z$-derivative image the high frequencies are amplified by a factor of $k$ making the transitions in $z$-derivative image sharper than those of the force image.

In summary the resolution is improved by decreasing the tip-to-sample distance, using a smaller tip and increasing the sensitivity of the scanning force microscope. The last limitation factor is often neglected.

Another important application of the present methods arises from the possibility to efficiently calculate stray fields of arbitrarily complex magnetization patterns. This allows one to adjust different parameters such as the tip-to-sample distance, the size of the sample magnetization, the tip magnetization, size or shape, or many other parameters to simulate an MFM image. As an example we use a Marquart fit routine to simulate an MFM image of the domain structure of a thin $\mathrm{Ni}$ film sandwiched between two $\mathrm{Cu}$ layers.

We use an molecular beam epitaxy (MBE) grown, epitaxial $\mathrm{Cu} / \mathrm{Ni} / \mathrm{Cu} / \mathrm{Si}(001)$ film with a $\mathrm{Ni}$ thickness of $10 \mathrm{~nm}$ and a capping layer of $2 \mathrm{~nm}$ thickness. The $\mathrm{Cu} / \mathrm{Ni} / \mathrm{Cu} /$ $\mathrm{Si}(001)$ system shows an extremely broad thickness range $(2-14 \mathrm{~nm})$ over which perpendicular magnetization is observed. ${ }^{13}$ A series of such films had previously been studied by MFM to reveal the dependence of the domain structure on the thickness of the Ni layer $(2-12.5 \mathrm{~nm})^{12}$ and to explain the transition of the magnetization direction from perpendicular to in-plane above a critical thickness. ${ }^{14} \mathrm{At}$ a Ni film thickness of $10 \mathrm{~nm}$, submicron bubble domains start to form inside micron sized domains [Fig. 9(a)] while the stability calculations show that the domain magnetization remains exactly perpendicular. ${ }^{72}$ Therefore the above theory can be applied.

Here we concentrate on the analysis of large scale images $\left(13.6 \times 15.8 \mu \mathrm{m}^{2}\right)$ where the magnetization pattern contains various spatial frequencies. The relatively large grid pitch of $53.1 \times 61.7 \mathrm{~nm}^{2}$ of the MFM image justifies the use of a simple force-transfer function [Eq. (22)]. We have tried both an extended dipole tip and a monopole (point charge) tip and found the latter to give better fit results [Fig. 4(b)]. This suggests that the top charge might be compensated by the usually complex domain structure on the pyramid tip. In the example shown in Fig. 9 we use the following procedure to simulate the MFM image [Fig. 9(c)] and to compare it to the MFM measurement [Fig. 9(a)]:

(1) We use the measured data to calculate a working image (not shown) at a reduced tip-to-sample distance [Fig. 9(a)]. Then we discriminate the working image at a certain level (1st fit parameter) to obtain the normalized (white $=1$, black $=-1$ ) magnetization pattern [Fig. 9(b).$^{93}$

(2) We calculate a force image [Fig. 9(c)] from the normalized magnetization pattern [Fig. 9(b)] using Eqs. (1), (8), (9), and (22) as a force-transfer function. The tip-tosample distance and the product of the tip charge and the sample magnetization are fit parameters.

(3) We use a Marquart-fit procedure to minimize the meansquare deviation of the difference of the simulated force image [Fig. 9(c)] and the measured MFM data [Fig. 9(a)]. The fit routine returns the three optimized parameters from which the first one, the level of the discrimination plane, has no physical value. The tip-to-sample distance becomes $176 \mathrm{~nm}$ which is larger than expected from approach curves. This reflects the fact that the magnetic tip (the point charge) is not located at the very end of the amorphous carbon needle probably because of the microscopic geometry (curvature) of the tip. The product of the charge of the tip and the magnetization of the sample becomes $1.66 \mathrm{nN}$. From the saturation magnetization of the sample $\left(M_{\mathrm{Ni}}=4.85 \times 10^{5} \mathrm{Am}^{-1}\right)$ we can calculate the point charge of the tip: $q_{\text {Tip }}=3.42$ 

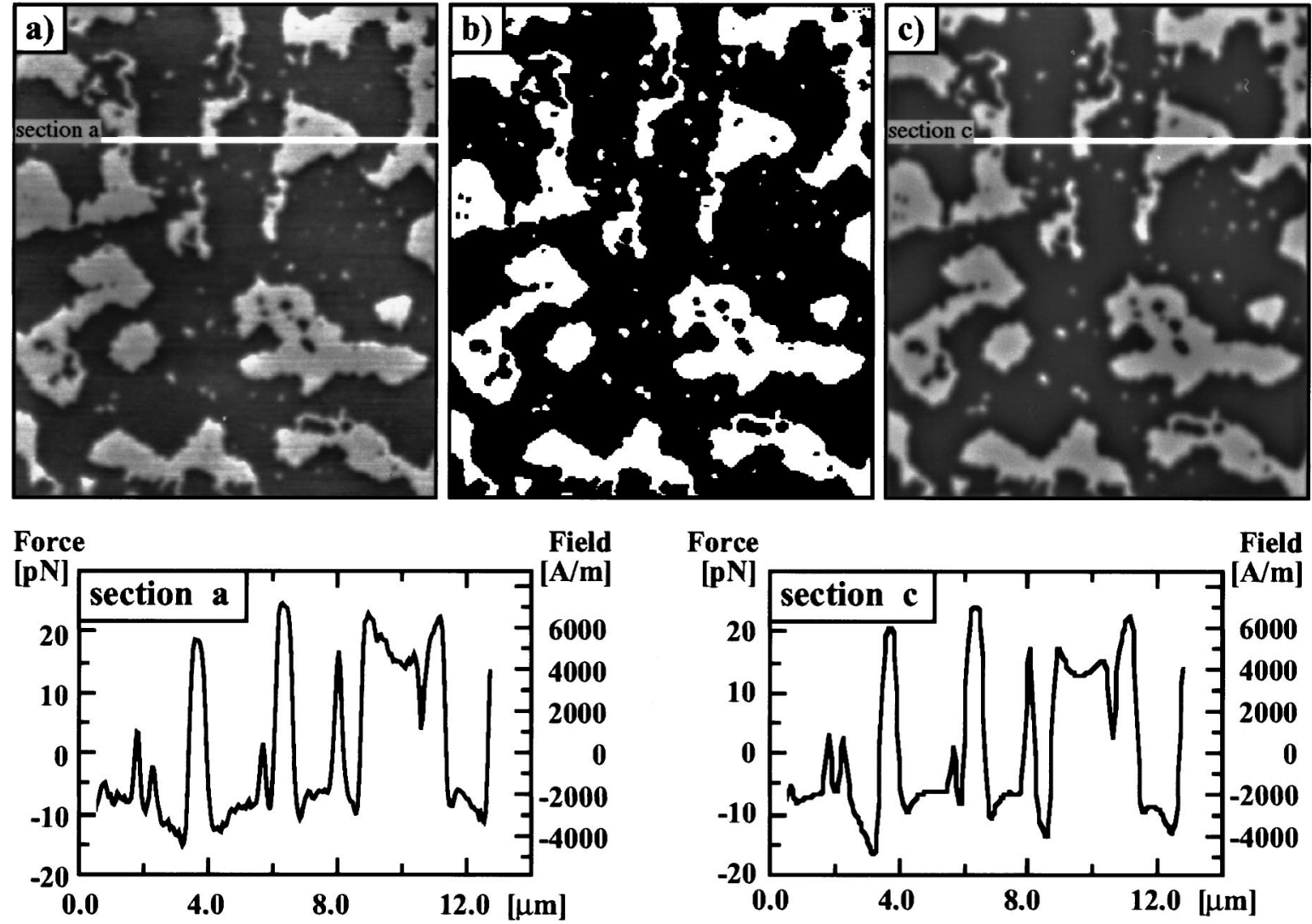

FIG. 9. Simulation of MFM data. (a) MFM measurement of a $\mathrm{Cu} / \mathrm{Ni}(10 \mathrm{~nm}) / \mathrm{Cu} / \mathrm{Si}(001)$ thin film showing perpendicular magnetization. (b) The magnetization pattern is generated by a discrimination process from the MFM measurement. (c) The simulation of the MFM image is generated from the magnetization pattern using the transfer function theory. (Sections a and c) Cross sections of the MFM measurement and the simulation.

$\times 10^{-6} \mathrm{nN} / \mathrm{Am}^{-1}$. From this value and the magnetization of the tip $\left(M_{\mathrm{Co}}=1.42 \times 10^{6} \mathrm{~A} / \mathrm{m}\right)$ we can further estimate [Eq. (22)] the bottom surface of the tip to be $1.91 \times 10^{-15} \mathrm{~m}^{2}$ or $20 \mathrm{~nm} \times 96 \mathrm{~nm}$. This suggests that the tip has about a 10:1 aspect ratio, which is consistant with Fig. 3.

Note that the above procedure can be used to calibrate the tip (evaluate the size of the point charge) on a standard sample with a well defined magnetization. Once the tip is calibrated, the size of the $z$ component of the stray field of a perpendicularly magnetized sample with an arbitrarily complex domain pattern can be measured quantitatively (see field axis in Fig. 9 sections a and c).

Thus the calibration of the tip has made it possible to assign a field value to the cross sectional force scans shown in Fig. 9. The question is, how consistent are these fields? The field for the $2.5 \mu \mathrm{m}$ domain, located around the $10 \mu \mathrm{m}$ position in section a), is $7 \times 10^{3} \mathrm{~A} / \mathrm{m}$ near the domain wall. This value is $1.4 \%$ of the saturation magnetization of $\mathrm{Ni}$. From Fig. 1(b) we can see that the ratio of the field to the magnetization (HTF) near the domain wall is in good agreement considering that the calculation was done in one dimension while the data are for a 2D-domain pattern. Further the calculation in Fig. 1(b) was done at a tip-to-sample distance of $100 \mathrm{~nm}$ while the magnetic charge-to-sample distance was determined to be $176 \mathrm{~nm} .{ }^{94}$ In addition to calculating a cor- rect and consistent magnitude for the field cross sections, their shapes are also in agreement with the predictions of transfer function theory. For example, the 600-nm-wide feature at the $6.5 \mu \mathrm{m}$ position in section a) of Fig. 9 gives the strongest stray field or force contrast. This is consistent with the dotted line in Fig. 1(b) for which the fundamental Fourier component occurs at the peak of the transfer function [dotted line in Fig. 1(a)]. The larger domain (2.5 $\mu \mathrm{m}$ wide) around the $10 \mu \mathrm{m}$ position generates the strongest stray field at the domain wall, while the interior of the domain produces a weaker field. This behavior is very well described by the dashed line of Fig. 1(b) which gives the field for a $2.5-\mu \mathrm{m}$ wide domain.

\section{OUTLOOK AND CONCLUSION}

In this article we have presented a transfer-function approach to calculate the stray field emanating from a perpendicularly magnetized sample and the force on a ferromagnetic MFM tip. The transfer-function method is easily implemented into today's image processing software packages which feature efficient handling of Fourier transformation of large matrices of real numbers. This allows the adjustment of different parameters such as the tip-to-sample distance, the size of the sample magnetization, the tip magnetization, size or shape, or many other parameters to simulate an MFM image. 
We have also described how and with what limitations the magnetization pattern can be calculated from the measured force image and how the sharpness of transitions can be enhanced. In addition we have mentioned the instrumental properties and operation modes required for quantitative magnetic force microscopy work. We have applied our theory to the analysis of magnetic force microscopy data acquired on a CoNi/Pt multilayer and on an epitaxially grown $\mathrm{Cu} / \mathrm{Ni} / \mathrm{Cu} / \mathrm{Si}(001)$ magnetic thin film.

Future theoretical work will include lateral magnetization structures and rough sample surfaces to allow fast stray field calculations of complex three-dimensional magnetization structures. Future experimental work will address the enhancement of the signal-to-noise ratio, the reduction of the size of the tip and the tip-to-sample distance in order to improve the lateral resolution of MFM measurements.

\section{APPENDIX}

It is instructive to derive the force on the MFM tip in direct space. We start from the total magnetostatic energy of the tip/sample system

$$
\begin{aligned}
E & =\frac{\mu_{0}}{2}\left[\int \mathbf{M}_{\mathrm{Tip}} \cdot \mathbf{H}_{\text {Sample }} d V+\int \mathbf{H}_{\mathrm{Tip}} \cdot \mathbf{M}_{\text {Sample }} d V\right] \\
& =\mu_{0} \int \mathbf{M}_{\text {Tip }} \cdot \mathbf{H}_{\text {Sample }} d V,
\end{aligned}
$$

where we have used the reciprocity principle to obtain Eq. (28) [the two integrals in Eq. (A1) are equal]. Note that the coordinate system is fixed to the surface of the tip and that the magnetization functions, $\mathbf{M}_{\text {Tip }}(x, y, z)$, and $\mathbf{M}_{\text {Sample }}(x, y, z)$, vanish outside the tip and sample volume, respectively. To calculate the force on the MFM tip it is useful to introduce a coordinate system, $\left(x^{\prime}, y^{\prime}, z^{\prime}\right)$, which is attached to the tip located at the position $\mathbf{t}=\left(t_{x}, t_{y}, t_{z}\right)$. Using the coordinates as defined in Fig. 2, Eq. (A2) can be written as

$$
E(\mathbf{t})=\mu_{0} \int \mathbf{M}_{\mathrm{Tip}}\left(x^{\prime}, y^{\prime}, z^{\prime}\right) \cdot \mathbf{H}_{\mathrm{Sample}}\left[\left(x^{\prime}, y^{\prime}, z^{\prime}\right)+\mathbf{t}\right] d V^{\prime} .
$$

The $z$ component of the force acting on the tip becomes

$$
\begin{aligned}
F_{z}(\mathbf{t})= & -\mu_{0} \int \mathbf{M}_{\mathrm{Tip}}\left(x^{\prime}, y^{\prime}, z^{\prime}\right) \\
& \cdot \frac{\partial}{\partial t_{z}} \mathbf{H}_{\text {Sample }}\left[\left(x^{\prime}, y^{\prime}, z^{\prime}\right)+\mathbf{t}\right] d V^{\prime} \\
= & -\mu_{0} \int \mathbf{M}_{\mathrm{Tip}}\left(x^{\prime}, y^{\prime}, z^{\prime}\right) \\
& \cdot \frac{\partial}{\partial z^{\prime}} \mathbf{H}_{\text {Sample }}\left[\left(x^{\prime}, y^{\prime}, z^{\prime}\right)+\mathbf{t}\right] d V^{\prime} .
\end{aligned}
$$

The other components of the force are easily evaluated by replacing the derivative in Eq. (A4) by the corresponding lateral derivatives.

It is noteworthy that for an extended tip the force is proportional to the integral of the derivative of the stray field and not proportional to the derivative of the field. For a comparison to the transfer-function approach we may assume the tip to be a long and thin, slablike object, with

(i) a bottom and a top surface, $\left(b_{x} b_{y}\right)$,

(ii) magnetized homogeneously along its long axis, $l$, and

(iii) oriented perpendicular to the surface of the sample (see Fig. 3).

Then Eq. (A4) becomes

$$
\begin{aligned}
F_{z}\left(t_{z}\right)= & -\mu_{0} M_{\text {Tip }} \cdot \int_{\left(b_{x} b_{y}\right)}\left[H_{z, \text { Sample }}\left(x^{\prime}+t_{x}, y^{\prime}+t_{y}, t_{z}\right)\right. \\
& \left.\left.-H_{z, \text { Sample }}\left(x^{\prime}+t_{x}, y^{\prime}+t_{y}, t_{z}+l\right)\right] d A^{\prime}, \quad \text { (A5 }\right)
\end{aligned}
$$

where the integration is evaluated only over the bottom and top surfaces of the tip (Fig. 4). The derivative along the $z$ direction of Eq. (A4) has become a difference (stray field at the bottom surface of tip minus stray field at the top surface of the tip) when the integration along the $z$ direction of Eq. (A4) is evaluated.

For a tip surface, $\left(b_{x} b_{y}\right)$, smaller than the grid resolution ${ }^{67}$ and a stray field decaying rapidly along the $z$ direction such that the stray field at the bottom surface is much larger than that at the top surface $\left[H_{z, \text { Sample }}\left(t_{z}\right)\right.$ $\left.\gg H_{z \text {,Sample }}\left(t_{z}+l\right)\right]$, Eq. (A5) finally becomes

$$
F_{z}(\mathbf{t})=q_{\text {Tip }} H_{z, \text { Sample }}(\mathbf{t}),
$$

where the tip is modeled by a magnetic point charge [Fig. 4(c) tip (c)], $q_{\text {Tip }}$, given by Eq. (22).

\section{ACKNOWLEDGMENTS}

The work at the Institute of Physics at the University of Basel was funded by the Swiss National Science Foundation and Basel Stadt. The work of G. Bochi and R. C. O'Handley at MIT was supported by NSF Grant No. DMR 94-10943. S. Porthun, L. Abelmann, and J. C. Lodder acknowledge the Dutch Stichting voor Fundamenteel Onderzoek der Materie (FOM) for their financial support of the work at MESA Research Institute.

${ }^{1}$ J. J. Saenz, N. Garcia, P. Grütter, E. Meyer, H. Heinzelmann, R. Wiesendanger, L. Rosenthaler, H. R. Hidber, and H.-J. Güntherodt, J. Appl. Phys. 62, 4293 (1987)

${ }^{2}$ Y. Martin and H. K. Wickramasinghe, Appl. Phys. Lett. 50, 1455 (1987).

${ }^{3}$ C. Schönenberger and S. F. Alvarado, Z. Phys. B 80, 373 (1990).

${ }^{4}$ H. J. Hug, Ph.D. thesis, Universität Basel, 1993.

${ }^{5}$ S. Porthun, Ph.D. thesis, University of Twente, 1996.

${ }^{6}$ A. Wadas, H. J. Hug, A. Moser, and H.-J. Güntherodt, J. Magn. Magn. Mater. 120, 379 (1992).

${ }^{7}$ A. Wadas, H. J. Hug, and H.-J. Güntherodt, Z. Phys. B 91, 403 (1993).

${ }^{8}$ J. R. Barnes, S. J. O'Shea, and M. E. Welland, J. Appl. Phys. 76, 418 (1994).

${ }^{9}$ R. Proksch, S. Foss, E. D. Dahlberg, and G. Prinz, J. Appl. Phys. 75, 5776 (1994).

${ }^{10}$ R. Proksch, S. Foss, C. Orme, S. Sahu, and B. Moskowitz, J. Appl. Phys. 75, 6892 (1994).

${ }^{11}$ D. E. Dahlberg, R. B. Proksch, B. M. Moskowitz, D. A. Bazylinski, and R. B. Frankel, J. Magn. Magn. Mater. 140-144, 1459 (1995).

${ }^{12}$ G. Bochi, H. J. Hug, D. I. Paul, B. Stiefel, A. Moser, I. Parashikov, H.-J. Güntherodt, and R. C. O'Handley, Phys. Rev. Lett. 75, 1839 (1995).

${ }^{13}$ G. Bochi, C. A. Ballentine, H. E. Inglefield, C. V. Thompson, R. C. O'Handley, H. J. Hug, B. Stiefel, A. Moser, and H.-J. Güntherodt, Phys. Rev. B 52, 7311 (1995). 
${ }^{14}$ H. J. Hug, G. Bochi, D. I. Paul, B. Stiefel, A. Moser, I. Parashikov, A. Klicznik, D. Lipp, H.-J. Güntherodt, and R. C. O'Handley, J. Appl. Phys. 79, 5609 (1996).

${ }^{15}$ Z. Quanmin, Y. Zheng, A. Roytburd, and M. Wuttig, Appl. Phys. Lett. 66, 2424 (1995).

${ }^{16}$ H. Hug, T. Jung, H.-J. Güntherodt, and H. Thomas, Physica C 175, 357 (1991).

${ }^{17}$ C. Bruder, Physica C 185, 1671 (1991)

${ }^{18}$ H. J. Reittu and R. Laiho, Supercond. Sci. Technol. 5, 448 (1992).

${ }^{19}$ A. Wadas, O. Fritz, H. J. Hug, and H.-J. Güntherodt, Z. Phys. B 88, 317 (1992).

${ }^{20}$ O. Fritz, M. Wülfert, A. Wadas, H. J. Hug, H.-J. Güntherodt, and H. Thomas, Phys. Rev. B 47, 384 (1993).

${ }^{21}$ H. J. Hug, A. Moser, O. Fritz, I. Parashikov, H.-J. Güntherodt, and Th. Wolf, Physica B 194-196, 377 (1994).

${ }^{22}$ H. J. Hug, A. Moser, I. Parashikov, B. Stiefel, O. Fritz, H.-J. Güntherodt, and H. Thomas, Physica C 235-240, 2695 (1994).

${ }^{23}$ A. Moser, H. J. Hug, I. Parashikov, B. Stiefel, O. Fritz, A. Baratoff, H. Thomas, H.-J. Güntherodt, and P. Chaudhari, Phys. Rev. Lett. 74, 1847 (1995).

${ }^{24}$ A. Wadas and H.-J. Güntherodt, J. Appl. Phys. 68, 4767 (1990).

${ }^{25}$ C. Schönenberger and S. F. Alvarado, J. Appl. Phys. 67, 7278 (1990).

${ }^{26}$ H. J. Hug, A. Moser, Th. Jung, A. Wadas, I. Parashikov, and H.-J. Güntherodt, Rev. Sci. Instrum. 64, 2920 (1993).

${ }^{27}$ A. Wadas, H. J. Hug, and H.-J. Güntherodt, J. Appl. Phys. 72, 203 (1992).

${ }^{28}$ G. Matteucci, M. Muccini, and U. Hartmann, Phys. Rev. B 50, 6823 (1994).

${ }^{29}$ G. Matteucci, M. Muccini, and U. Hartmann, IEEE Trans. Magn. 133, 422 (1994).

${ }^{30}$ T. Göddenhenrich, U. Hartmann, and C. Heiden, Ultramicroscopy 42-44, 256 (1992).

${ }^{31}$ T. Ohkubo, J. Kishigami, and K. R. Kaneko, NTT Res. and Dev. (Japan) 42, 545 (1993).

${ }^{32}$ T. Ohkubo, J. Kishigami, and K. R. Kaneko, IEEE Trans. Magn. 29, 4086 (1993).

${ }^{33}$ K. Wago, K. Sueoka, and F. Sai, IEEE Trans. Magn. 27, 5178 (1991).

${ }^{34}$ K. Sueoka, K. Wago, and F. Sai, IEEE Trans. Magn. 28, 2307 (1992).

${ }^{35}$ O. Watanuki, F. Sai, and K. Sueoka, Ultramicroscopy 42-44, 315 (1992).

${ }^{36}$ S. Takayama, K. Sueoka, H. Setoh, R. Schafer, B. E. Argyle, and P. L. Trouilloud, IEEE Trans. Magn. 28, 2647 (1992).

${ }^{37}$ G. A. Gibson, S. Schultz, T. Carr, and T. Jagielinski, IEEE Trans. Magn. 28, 2310 (1992).

${ }^{38}$ G. Persch and H. Strecker, Ultramicroscopy 42-44, 1269 (1992).

${ }^{39}$ A. Hubert, Institut für Werkstoffwissenschaften Universität Erlangen, Martenstrasse 7 D-91058 Erlangen, Schluchsee, Germany, 1994 (private communication).

${ }^{40}$ J. Saenz and N. Garcia, J. Appl. Phys. 63, 2947 (1988).

${ }^{41}$ U. Hartmann, J. Appl. Phys. 64, 1561 (1988).

${ }^{42}$ U. Hartmann and C. Heiden, J. Microsc. 152, 281 (1988).

${ }^{43}$ U. Hartmann, Phys. Rev. B 40, 7421 (1989).

${ }^{44}$ A. Wadas, J. Magn. Magn. Mater. 78, 263 (1989).

${ }^{45}$ A. Wadas and P. Grütter, Phys. Rev. B 39, 12013 (1989).

${ }^{46}$ A. Wadas and H.-J. Güntherodt, Phys. Lett. A 146, 277 (1990).

${ }^{47}$ A. Wadas, P. Grütter, and H.-J. Güntherodt, J. Vac. Sci. Technol. A 8, 416 (1990).

${ }^{48}$ A. Wadas, P. Grütter, and H.-J. Güntherodt, J. Appl. Phys. 67, 3462 (1990).

${ }^{49}$ U. Hartmann, J. Vac. Sci. Technol. A 8, 411 (1990).

${ }^{50}$ D. Rugar, H. J. Mamin, P. Güthner, P. Lambert, J. E. Stern, I. McFadyen, and T. Yogi, J. Appl. Phys. 68, 1169 (1990).

${ }^{51}$ A. Mansuripur and R. Giles, IEEE Trans. Magn. 25, 3467 (1989).

${ }^{52}$ M. Scheinfein, J. Unguris, D. Pierce, and R. Celotta, J. Appl. Phys. 67, 5932 (1990).

${ }^{53}$ A. Aharoni and J. P. Jakubovics, J. Appl. Phys. 73, 6498 (1993).

${ }^{54}$ J. O. Oti, IEEE Trans. Magn. 29, 2359 (1993).

${ }^{55}$ J. O. Oti and P. Rice, J. Appl. Phys. 73, 5802 (1993).

${ }^{56}$ S. Müller-Pfeiffer, M. Schneider, and W. Zinn, Phys. Rev. B 49, 15745 (1994).

${ }^{57}$ Note that a transfer function theory is also possible in the case of a MFM tip above a superconductor in the Meissner state (soft sample) (Ref. 16).

${ }^{58}$ C. Xiaodong, M. Lederman, G. A. Gibson, H. N. Bertram, and S. Schultz, J. Appl. Phys. 73, 5805 (1993).

${ }^{59}$ I. D. Mayergoyz, A. A. Adly, R. D. Gomez, and E. R. Burke, J. Appl. Phys. 73, 5799 (1993).
${ }^{60}$ T. Chang, M. Lagerquist, J.-G. Zhu, J. H. Judy, P. B. Fischer, and S. Y. Chou, IEEE Trans. Magn. 28, 3138 (1992).

${ }^{61}$ C. D. Wright and E. W. Hill, Appl. Phys. Lett. 68, 1726 (1996).

${ }^{62}$ Here we treat only perpendicularly magnetized samples. However, the application of transfer functions to an arbitrarily complex micromagnetic structure including in-plane magnetization components is straight forward.

${ }^{63} \sigma=\mathbf{M}_{\text {Sample }} \cdot \mathbf{n}$ with $\mathbf{n}$ being the surface normal.

${ }^{64}$ C. Kittel, Phys. Rev. 70, 945 (1946)

${ }^{65}$ C. Kooy and U. Enz, Philips Res. Rep. 15, 7 (1960).

${ }^{66}$ Note that the force exerted from the tip onto the sample is now easily calculated by a multiplication of Eq. (16) times $2 \mu_{0}$ with the distance loss factor, $e^{-k z}$, and integration over the sample charge distribution [reciprocity principle, Ref. 61].

${ }^{67}$ These zeros are analogous to the gap null in a magnetic recording head.

${ }^{68} \mathrm{H}$. N. Bertram, Theory of Magnetic Recording 4 (Cambridge University Press, Cambridge, 1994).

${ }^{69}$ The cutoff frequency of the tip is determined by the first root of the force-transfer function. In the case of the parallel piped tip Eq. (21), the critical wavelength, $\lambda_{\text {crit }}$ is equal to size of the bottom surface of the tip, $b_{x}$ or $b_{y}$.

${ }^{70}$ Visual Numerics Inc., PV-WAVE, 6230 Lookout Road, Boulder Colorado 80301.

${ }^{71}$ R. Hofer, B. E. Bürgler, G. Tarrach, and D. Brodbeck, SXM-Shell, Institute of Physics, University of Basel, Klingelbergstrasse 82, CH-4056 Basel, Switzerland (unpublished).

${ }^{72}$ H. J. Hug et al., 1996 (unpublished).

${ }^{73}$ R. Wiesendanger and H.-J. Güntherodt, Scanning Tunneling Microscopy II (Springer, Germany, 1992), Vol. Springer Series in Surface Science 28.

${ }^{74}$ Note that this is a particular problem with microfabricated $\mathrm{SiN}_{4}$ cantilevers. Due to their low mass, the energy stored in the cantilever oscillation is small and the damping caused by air flowing in and out the gap formed by the relatively flat pyramid tip and the surface of the sample becomes severe.

${ }^{75}$ Note that this is also true for all present true-atomic-resolution experiments.

${ }^{76}$ The temperature plays an important role. Measurements performed at $3 \mathrm{~K}$ instead of room temperature will improve the minimal detectable force derivative by a factor of ten. Further the decreased drift rate at low temperatures allows long measurement times and therefore reduced measurements bandwidths, $\Delta \nu$ leading to improved force sensitivity.

${ }^{77}$ F. J. Giessible, Science 267, 68 (1995).

${ }^{78}$ P. Güthner, J. Vac. Sci. Technol. B 14, 2428 (1995).

${ }^{79}$ S. Kitamura and M. Iwatsuki, Jpn. J. Appl. Phys., Part 1 34, 145 (1995).

${ }^{80}$ H. Ueyama, M. Ohta, Y. Sugawara, and S. Morita, Jpn. J. Appl. Phys., Part 1 34, 1086 (1995).

${ }^{81}$ R. Luethi, E. Meyer, M. Bammerlin, A. Baratoff, T. Lehmann, L. Howald, Ch. Gerber, and H.-J. Güntherodt, Z. Phys. B 100, 165 (1996).

${ }^{82}$ S. Kitamura and M. Iwatsuki, Jpn. J. Appl. Phys., Part 1 35, 668 (1996).

${ }^{83}$ L. Olsson, R. Wigren, and R. Erlandson, Rev. Sci. Instrum. 67, (1996).

${ }^{84}$ T. R. Albrecht, P. Grütter, D. Horne, and D. Rugar, J. Appl. Phys. 69, 668 (1991).

${ }^{85}$ H. J. Hug, A. Moser, D. Weller, I. Parashikov, A. Tonin, H. R. Hidber, and H.-J. Güntherodt, Physica B 12, 1591 (1994).

${ }^{86}$ M. Rührig, S. Porthun, and J. C. Lodder, Rev. Sci. Instrum. 165, 3225 (1994).

${ }^{87}$ S. Y. Chou, M. S. Wei, and P. B. Fischer, IEEE Trans. Magn. 30, 4485 (1994).

${ }^{88}$ M. Rührig, S. Porthun, J. C. Lodder, S. McVitie, L. J. Heyderman, A. B. Johnston, and J. N. Chapman, J. Appl. Phys. 79, 2913 (1996).

${ }^{89}$ A. Moser, H. J. Hug, Th. Jung, U. D. Schwarz, and H.-J. Güntherodt, Meas. Sci. Technol. 4, 769 (1993).

${ }^{90}$ CAMST Topical Meeting on Magnetic Force Microscopy (MESA Research Institute, University of Twente, The Netherlands, 1995).

${ }^{91}$ A similar procedure would be applicable to scanning tunneling microscopy data (exponential decay of the corrugation above a periodic atomic structure) as well as to scanning near field optical microscopy data (exponential decay of the evanescent light field of a standing surface wave).

${ }^{92}$ A. J. Meixner, M. A. Bopp, and G. Tarrach, Appl. Opt. 33, 7995 (1994).

${ }^{93}$ Note that many of the small domains would be missed by the discrimination plane if the original data is used instead of the working image with its reduced tip-to-sample distance. However the exact amount of the reduc- 
tion and the assumed tip-to-sample distance of the measured data are not sensitive parameters. Here we assume the tip-to-sample distance of the measured data to be $170 \mathrm{~nm}$ and the reduced tip-to-sample distance to be $150 \mathrm{~nm}$.
${ }^{94}$ The magnetic charge-to-sample distance of $176 \mathrm{~nm}$ is larger than the distance between the physical end of the tip and the sample $(\approx 100 \mathrm{~nm})$ as determined from the experiment. This is reasonable given the radius of curvature of the tip $(50-100 \mathrm{~nm})$. 